\title{
Retrograde Axonal Transport of Endogenous Phospholipids in Rat Sciatic Nerve ${ }^{1}$
}

\author{
REGINA ARMSTRONG, ${ }^{\star} \ddagger$ ARREL TOEWS, ${ }^{\star} \S$ RITA B. RAY, ${ }^{*}$ AND PIERRE MORELL ${ }^{\star} \ddagger \$^{2}$ \\ * Biological Sciences Research Center, $¥$ Curriculum in Neurobiology, and §Department of Biochemistry and Nutrition, University of \\ North Carolina, Chapel Hill, North Carolina 27514
}

\begin{abstract}
Anterograde axonal transport of phospholipids occurs at a rate of several hundred millimeters per day. However, although labeled precursors are incorporated into phospholipids in the neuronal cell bodies within several hours, these newly synthesized phospholipids are committed to transport over a much longer period of time. Thus, maximal accumulation of radioactive lipids in axons and nerve endings does not occur for several days (e.g., 4 to 7 days in rat optic tract and sciatic nerve). We have now investigated the retrograde axonal transport of endogenous phospholipid molecules in sensory neurons of rat sciatic nerve. Labeled phospholipids were delivered to axons and nerve endings of these cells by anterograde axonal transport following injection of $\left[2-{ }^{3} \mathrm{H}\right]$ glycerol into the $L 5$ dorsal root ganglion. At various times following precursor injection two ligatures, $9 \mathrm{~mm}$ apart, were applied to the mid-thigh region of the sciatic nerve. Animals were sacrificed 3 to $\mathbf{4 8} \mathrm{hr}$ after nerve ligation, nerves were dissected and sectioned into 5 - $\mathrm{mm}$ segments, and phospholipid radioactivity in each segment was determined. The timedependent accumulation of labeled phospholipids distal to the distal ligature demonstrated their retrograde axonal transport. The time course of retrograde transport for these phospholipids was more prolonged and peaked several days later than the time course for the anterograde transport phase.
\end{abstract}

Further information regarding the relationship between radioactive phospholipids arriving at the nerve endings by anterograde transport, and their subsequent "turn-around" and retrograde transport back to the nerve cell bodies, was obtained by analyzing the phospholipid class label distribution of both of these transport phases at various times following precursor injection. Labeled lipids initially returning to the neuronal cell bodies by retrograde transport were enriched in phosphatidylcholine. This presumably reflects the fact that labeled phospholipids initially reaching the nerve endings by anterograde transport are also enriched in phosphatidylcholine (Toews, A. D., B. F. Saunders, W. D. Blaker, and P. Morell (1983) J. Neurochem. 40: 555-562). Subsequent temporal

Received June 4, 1984; Revised September 17, 1984;

Accepted September 24, 1984

\footnotetext{
${ }^{1}$ This work was presented at the 15th Annual Meeting of the American Society for Neurochemistry (Armstrong et al., 1984). This research was supported by United States Public Health Service Grants NS11615 and HDO3110.

${ }^{2}$ To whom correspondence and reprints requests should be addressed at Biological Sciences Research Center $220 \mathrm{H}$, University of North Carolina, Chapel Hill, NC 27514.
}

changes in the distribution of radioactivity among individual phospholipid classes in the retrograde transport phase were similar to those seen for anterograde transport but were delayed by several days. These findings suggest that, as lipids arrive at axons and nerve endings by anterograde transport, they enter a pool of lipids from which they are available for incorporation into the retrograde phase of axonal transport.

The intracellular transport of material along axons has been extensively investigated (for reviews, see Schwartz, 1979; Grafstein and Forman, 1980; Ochs, 1983; and a recent monograph, Weiss, 1982). The anterograde axonal transport of protein molecules, synthesized in the neuronal cell bodies, involves several compositionally distinct phases, each with a characteristic transport rate (Karlsson and Sjostrand, 1971; Willard et al., 1974; Hoffman and Lasek, 1975; Levine and Willard, 1980; Tytell et al. 1981). Rapidly transported proteins are associated mainly with particulate material (for example, see Cuenod and Schonbach, 1971; Elam and Agranoff, 1971; Di Giamberardino et al., 1973; Sjostrand, 1979), primarily vesiculotubular structures (Droz et al., 1975; Rambourg and Droz, 1980; Tsukita and Ishikawa, 1980).

Phospholipids are also synthesized in the neuronal cell bodies and, subsequently, some are subjected to anterograde axonal transport. This has been demonstrated in peripheral nerves (Miani, 1962; Abe et al., 1973; Alberghina et al., 1983; Toews et al., 1983), the visual system (Grafstein et al., 1975; Currie et al., 1978; Haley et al. 1979; Toews et al., 1979; Alberghina et al., 1981), and other CNS tracts (Toews et al., 1980). Radioactive precursors are rapidly incorporated into newly synthesized phospholipids, which are then conveyed to the axons and nerve endings in the rapid phase of anterograde axonal transport, presumably in the form of membranous material (Graftstein, 1975; Longo and Hammerschlag, 1980). However, in contrast to proteins, commitment of the newly labeled pool of phospholipid to transport occurs over several days (Graftstein et al., 1975; Haley et al., 1979; Toews et al., 1979, 1983). During anterograde transport the phospholipids may exchange between axonal organelles or transfer into glial membranes or myelin sheaths (Brunetti et al., 1981; Droz et al., 1981; Gould et al., 1982; Ledeen and Haley, 1983).

Retrograde axonal transport has also been demonstrated for protein molecules and for various exogenous macromolecules (for reviews, see Thoenen and Schwab, 1978; Bisby, 1980, 1982; Kristensson, 1982; Schwab and Thoenen, 1983). Membranous structures of vesicular, multivesicular, vacuolar, and tubular forms appear to be involved in the return of this material to the nerve cell body (Kapeller and Mayor, 1969; Forman et al. 1977; Smith, 1980; Tsukita and Ishikawa, 1980). The retrograde movement of lipids can be inferred from these observations, since these membranous structures presumably must contain lipids. 
We have now investigated directly the kinetics of retrograde axonal transport of endogenous phospholipids in the sensory neurons of the rat sciatic nerve. Phospholipids in the axons and in the nerve endings were labeled by anterograde axonal transport, and the subsequent retrograde transport of these labeled lipids was examined using a double ligature technique.

\section{Materials and Methods}

Injection of radioactive precursors. Adult male Long-Evans rats were injected subcutaneously with $1.0 \mathrm{ml}$ of atropine sulfate $(0.4 \mathrm{mg} / \mathrm{ml})$ as an anesthetic pretreatment to block bronchial secretions. Fifteen minutes later, the animals were placed under ketamine/xylazine anesthesia $(87$ and $13 \mathrm{mg} /$ $\mathrm{kg}$ of body weight, respectively) administered intramuscularly into the forelimb. The spinal column was stabilized at the pelvis in a stereotaxic apparatus (David Kopf Instruments, Tujunga, CA), and a dorsal laminectomy was performed to expose both L5 dorsal root ganglia. A solution containing 200 $\mu \mathrm{Ci} / \mu \mathrm{l}$ of $\left[2{ }^{3} \mathrm{H}\right] \mathrm{glycerol}(10 \mathrm{Ci} / \mathrm{mmol}$; New England Nuclear, Boston, MA) was prepared in $0.9 \% \mathrm{NaCl}$ (tinted with chlorophenyl red dye to aid in monitoring the progress of the injection). This solution was drawn into a calibrated capillary micropipette (inside and outside diameter of tip approximately 13 and $30 \mu \mathrm{m}$, respectively) secured in a micromanipulator. One microliter of solution was injected into each L5 ganglion over a 30- to 60-sec period, using pressure applied via an air-filled $12-\mathrm{ml}$ syringe. Following the injection, the micropipette was left in place for $90 \mathrm{sec}$ and then withdrawn. The wound bed was then flushed with $0.9 \% \mathrm{NaCl}$ and packed with sterile Gelfoam before closure with wound clips.

Nerve ligation and dissection. At intervals of $12 \mathrm{hr}$ to 21 days from the injection time, animals were again placed under ketamine/xyiazine anesthesia. The left sciatic nerve was exposed in the mid-thigh area and two 5-0 silk ligatures were applied $9 \mathrm{~mm}$ apart, approximately $40 \mathrm{~mm}$ and $50 \mathrm{~mm}$ distal to the injected ganglion. Animals were sacrificed 6 to $48 \mathrm{hr}$ atter nerve ligation. The left and right dorsal root ganglia and sciatic nerves were then dissected and sectioned into $5-\mathrm{mm}$ segments. The $9-\mathrm{mm}$ length of nerve between the ligatures was cut into three 3 -mm segments. All segments were freeze-dried and stored trozen until analysis.

Analysis of tissue samples. Lipids were extracted from the freeze-dried tissue samples using a modification (Benjamins et al., 1976) of the method of Folch et al. (1957). Total lipid radioactivity was determined by counting an aliquot of the lipid extract in a liquid scintillation spectrometer. Phospholipid classes were isolated from the remainder of the lipid extracts by twodimensional thin-layer chromatography with an intermediate acid hydrolysis step (Horrocks and Sun, 1972). Individual lipid classes were visualized by exposure to iodine vapor and scraped into scintillation vials. After addition of $0.4 \mathrm{ml}$ of water and $10 \mathrm{ml}$ of a Triton X-100-based scintillation mixture (Miller et al., 1975), radioactivity was determined by liquid scintillation counting

Calculation of relative accumulation. Due to experimental variables (primarily precursor injection variability) and a rather steep proximodistal gradient of radioactivity along the nerves, the unligated contralateral nerve did not always provide an adequate estimate of a base line necessary for calculating accumulation in a ligated nerve segment. Therefore, accumulation of lipid radioactivily at a ligature was calculated by the method of Bisby (1977), as shown in Figure 1. This provides a measure of increased lipid radioactivity in the accumulation segment relative to estimated base line radioactivity. The estimated base line is an averaged level of lipid radioactivity per millimeter in segments of approximately equal length on both sides of the accumulation segment. Thus, if the accumulation segment has twice as many disintegrations per minute per millimeter as the average number of disintegrations per minute per millimeter in the two bracketing segments, the relative accumulation value is 2.0. These relative accumulation values reflect the amount of labeled material being transported in each direction during the time interval between nerve ligation and dissection (they do not reflect the absolute amount of transported material deposited in axons, since this is controlled for by use of the bracketing segments to define the base line). Measurement of accumulation of phospholipid radioactivity at each ligature may actually underestimate the amount of transport, since there may be some local "turnaround" of transported material at the ligature (Bisby and Bulger, 1977).

\section{Results}

Dependence of lipid accumulation on ligature time. The maximum accumulation of axonally transported phospholipid radioactivity in axons and nerve terminals occurs 4 to 7 days after precursor injection (Toews et al., 1983). Therefore, a 7-day transport time (period from precursor injection until ligatures were applied) was chosen as the starting point for studying retrograde transport. Virtually all $(>95 \%)$ of the lipid label present along the nerve is in phospholipids after injection of $\left[2^{3} \mathrm{H}\right]$ glycerol (Toews ct al., 1983), presumably because this precursor is poorly reutilized (Benjamins and McKhann, 1973). The time dependence of accumulation of labeled transported lipids at the ligatures was examined at collection times (period from ligature application until animals were sacrificed) varying from 6 to $48 \mathrm{hr}$. Examples from two collection times are shown in Figure 2. Lipid radioactivity above that in the bracketing segments was present in both the retrograde and anterograde accumulation segments. Between 6 and $24 \mathrm{hr}$, there was a considerable increase in label in both the retrograde and anterograde accumulation segments. Levels of lipid radioactivity in the retrograde accumulation segment were $30 \%$ greater than the estimated base line values at $6 \mathrm{hr}$ and $88 \%$ greater at $24 \mathrm{hr}$. The continuous increase in the retrograde relative accumulation value with increasing collection time (Fig. 3 ) indicates that labeled phospholipids en route back to the nerve cell bodies accumulated distal to the distal ligature in a time-dependent manner.

Time course of retrograde axonal transport. The time course of retrograde axonal transport of phospholipids was examined as a function of time allowed for anterograde transport and was compared to the time course of anterograde transport. Transport time was varied between $12 \mathrm{hr}$ and 21 days to examine the time course of accumulation of labeled lipids at each ligature. Since there was substantial accumulation of labeled lipids with a 24 -hr collection
Figure 1. Schematic diagram of sciatic nerve illustrating the model used to examine anterograde and retrograde axonal transport, and the method used to calculate relative accumulation. Each accumulation segment is $5 \mathrm{~mm}$ in length, and the segment between the ligatures is $9 \mathrm{~mm}$ long. The proximal and distal bracketing segments are each $10 \mathrm{~mm}$.

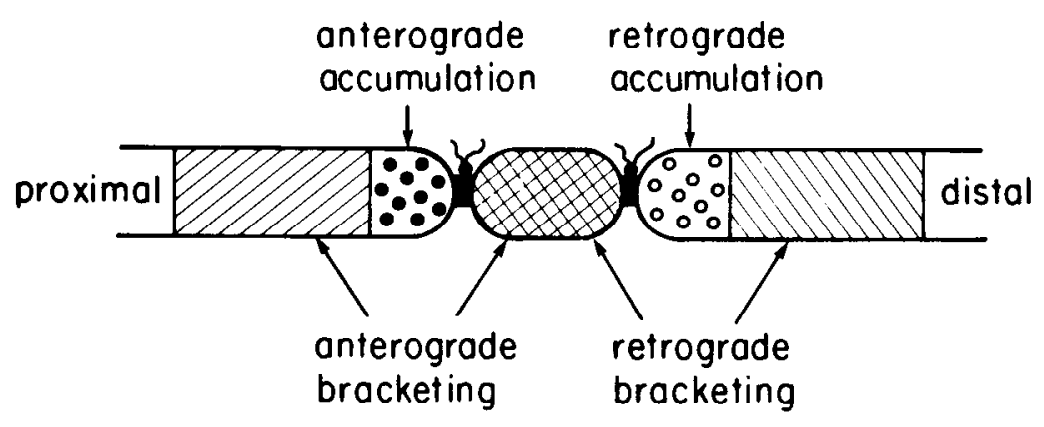

RELATIVE ACCUMULATION $=\frac{\text { lipid radioactivity } / \mathrm{mm} \text { in accumulation segment }}{\text { lipid radioactivity } / \mathrm{mm} \text { in estimated baseline }}$ 

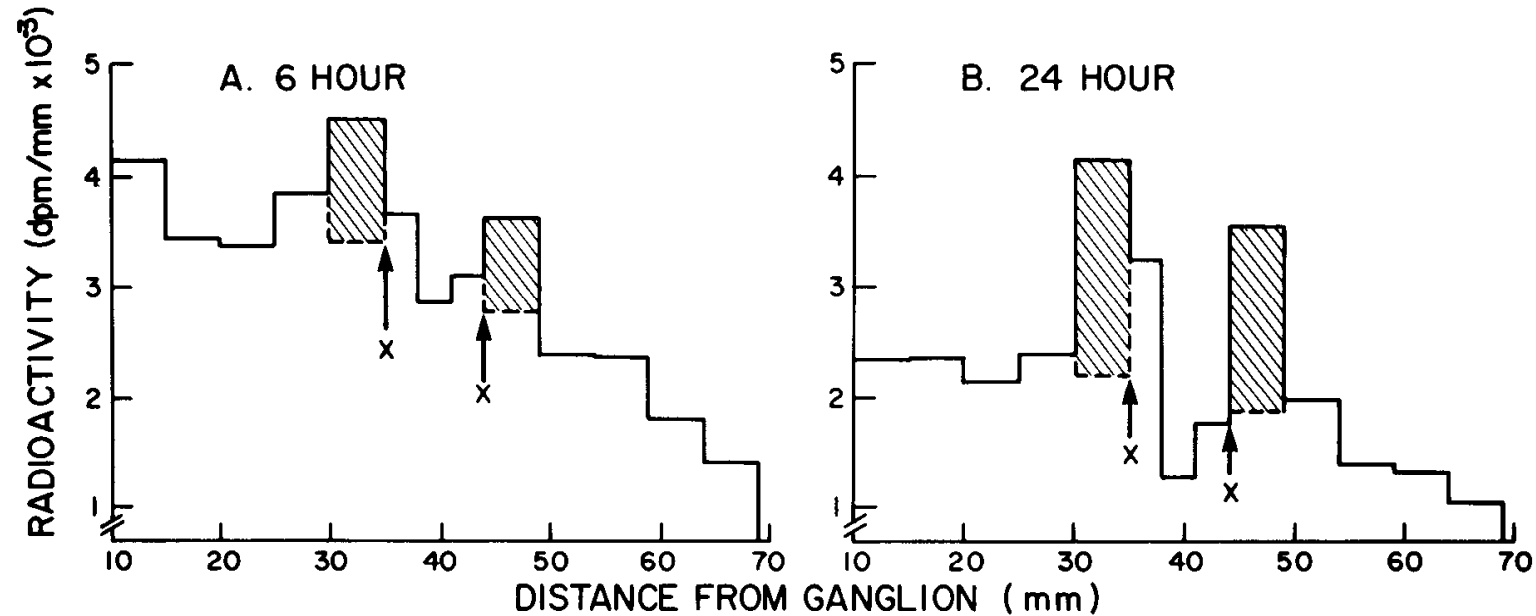

Figure 2. Patterns of lipid radioactivity in ligated sciatic nerve. Ligatures were applied 7 days after injection of precursor into the $L 5$ dorsal root ganglion and were left in place for $6 \mathrm{hr}(A)$ or $24 \mathrm{hr}(B)$ before animals were sacrificed. A cross-hatched pattern indicates increased lipid radioactivity in the anterograde and retrograde accumulation segments relative to their respective estimated base line values, which were calculated as shown in Figure 1 . The estimated base line is an averaged level of radioactivity per millimeter in segments of approximately equal length on both sides of the accumulation segments. Between 6 and $24 \mathrm{hr}$ there was a considerable increase in label in both the retrograde and anterograde accumulation segments.

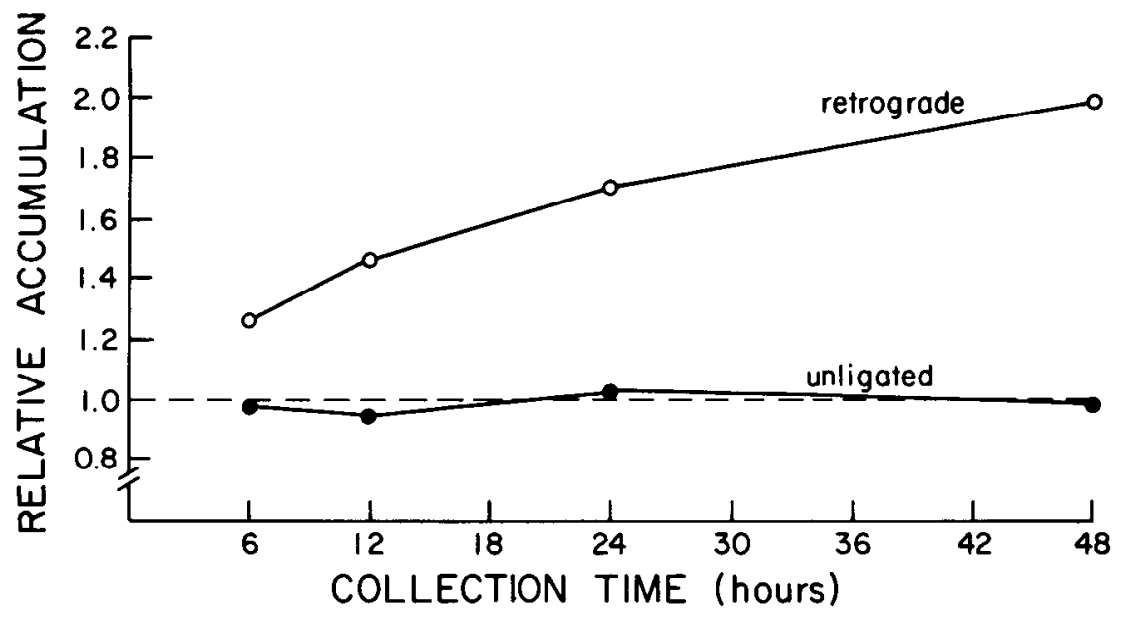

Figure 3. Dependence of accumulation of retrogradely transported phospholipids on collection time. $\left[2-{ }^{3} \mathrm{H}\right]$ Glycerol was injected into both $\mathrm{L} 5$ dorsal root ganglia, and the axons and nerve endings were allowed to accumulate labeled macromolecules by axonal trans port for 7 days (transport time). Ligatures were then applied to the left sciatic nerve and were left in place for times ranging from 6 to $48 \mathrm{hr}$ (collection time) before animals were sacrificed. $O$, relative accumulation values for the retrograde accumulation segment (segment distal to the distal ligature); $\boldsymbol{\theta}$, relative accumulation values for the corresponding segment from the right (unligated) nerve. Relative accumulation values for the retrograde accumulation segment increased with increasing collection time, demonstrating the time-dependent accumulation of labeled phospholipids en route back to the nerve cell bodies. Relative accumulation values for the unligated control segment were all very close to one, as expected in the case of no accumulation.

period (Fig. 3), this interval was chosen as the uniform accumulation period for the time course studies.

Relative accumulation values (Fig. 4) reflect the time course of labeled phospholipid movement along the nerve. The peaks indicate the period of the maximal rate of accumulation of radioactive lipids at each ligature. The maximal retrograde accumulation (4 to 10 days) occurs several days later and is more prolonged than that for anterograde accumulation ( 1 to 3 days). A control for methodological variability is that relative accumulation values in corresponding unligated segments in the contralateral injected nerve were very close to one. This is as expected for the case of no accumulation.

In a control experiment, a set of four animals was injected in only one $\mathrm{L} 5$ dorsal root ganglion, and the contralateral ganglion and nerve were analyzed for systemic background labeling due to isotope leakage and diffusion from the injection site. Levels of lipid radioactivity in the contralateral nerve segments were usually less than $1 \%$ of the levels present in corresponding segments from the injected nerve, indicating that systemic background labeling was not significant. Thus, levels of lipid radioactivity in the nerve reflect axonally transported phospholipids.

Temporal changes in phospholipid class radioactivity distribution for lipids undergoing anterograde and retrograde transport. Timedependent changes in the distribution of radioactivity among the major phospholipid classes were examined in the retrograde accumulation segment following thin-layer chromatography (Fig. 5). Anterograde label distribution changes were also determined, using an unligated contralateral nerve segment which was of equal distance from the ganglion as the retrograde accumulation segment. Collection periods were $24 \mathrm{hr}$, except for the two earliest time points, when a 12-hr collection interval was used to provide a more sensitive measure of the early material committed to retrograde transport.

Phosphatidylcholine and phosphatidylethanolamine were the major labeled lipids transported in the retrograde direction, together accounting for 60 to $80 \%$ of the total accumulating radioactivity. However, the relative proportion of label in these two lipid classes changed with transport time (Fig. 5). In the retrograde accumulation segment, the ratio of radioactive phosphatidylcholine to phosphatidylethanolamine was higher at early time points, relative to later times, indicating an initial enrichment of labeled phosphatidylcholine and a slight depletion of labeled phosphatidylethanolamine. These label distribution changes seen for retrogradely transported lipids were similar to changes seen several days earlier in the anterograde phase of transport. Phosphatidylserine and phosphatidylinositol accounted for most of the remaining lipid radioactivity present in the retrograde accumulation segment.

\section{Discussion}

Endogenous phospholipids delivered to axons and nerve endings by anterograde axonal transport subserve various functions, including serving as structural components in nerve ending membranes and synaptic vesicles. These phospholipids eventually turn over with apparent half-lives ranging from days to several weeks (Toews and 


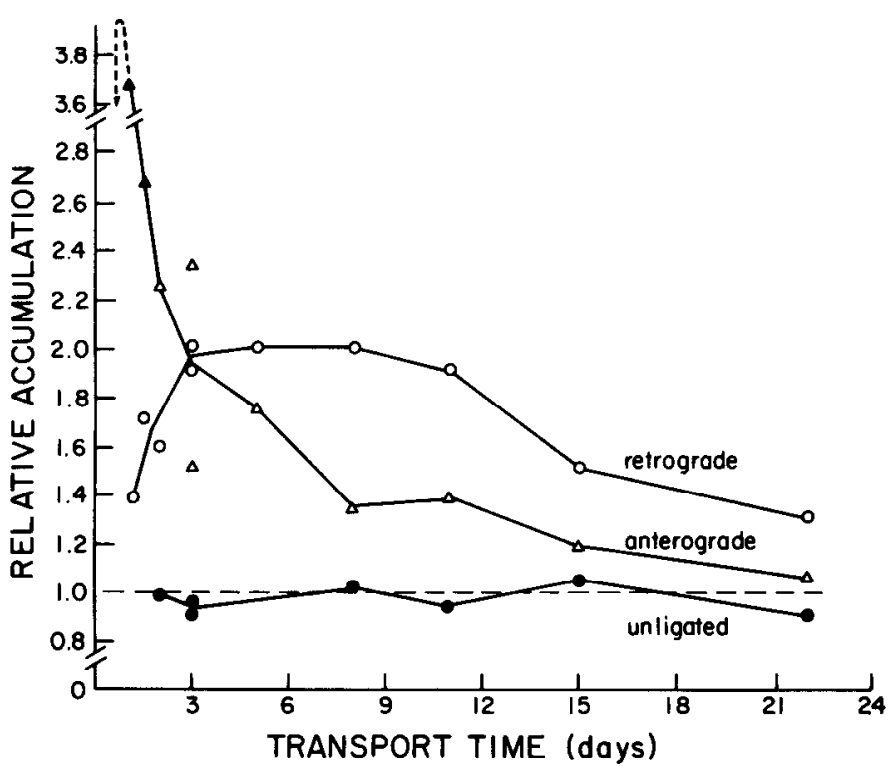

Figure 4. Time courses of anterograde and retrograde axonal transport of phospholipids as a function of transport time. $\left[2-{ }^{3} \mathrm{H}\right]$ Glycerol was injected into both $\mathrm{L} 5$ dorsal root ganglia, and at times ranging from $12 \mathrm{hr}$ to 21 days after injection (transport time), ligatures were placed on the left sciatic nerve. $\Delta$, relative accumulation values for anterograde accumulation segment (segment proximal to the proximal ligature); $O$, relative accumulation values for retrograde accumulation segment (segment distal to the distal ligature); $\boldsymbol{\bullet}$, relative accumulation values for the segment in the contralateral (unligated) nerve corresponding to the retrograde accumulation segment. All collection times were $24 \mathrm{hr}$ except the first two points of the retrograde and anterograde lines, which were obtained from 12-hr collection times. Relative accumulation peaks reflect periods of maximal accumulation of radioactive phospholipids at each ligature. The maximal retrograde accumulation (4 to 10 days) occurs several days later and is more prolonged than that for anterograde accumulation ( 1 to 3 days). No accumulation was seen in the control segment from the unligated contralateral nerve.

Morell, 1981; Toews et al., 1983). Although some of these lipids may actually be hydrolyzed enzymatically in axons and nerve endings, much of the removal of these radioactive lipids presumably involves return to the nerve cell bodies. This aspect of neuronal metabolism has been well documented for endogenous proteins (for review, see Thoenen and Schwab, 1978; Bisby, 1980, 1982; Kristensson, 1982; Schwab and Thoenen, 1983). During the retrograde phase of transport phospholipids may have several functional roles. Phospholipids provide the lipid environment which allows for the retrograde transport of endogenous proteins. They must also comprise part of the endocytic vesicles which engulf exogenous molecules and particles at nerve endings for transport back to the cell body (for review, see Schwab and Thoenen, 1983).

In this study, we utilized anterograde axonal transport to initially label endogenous phospholipids in axons and nerve endings of sciatic nerve sensory neurons. The subsequent time-dependent accumulation of labeled phospholipids distal to a ligature around the nerve demonstrates the retrograde axonal transport of endogenous phospholipids (see also a preliminary report by Bisby, 1983). Although newly synthesized phospholipids are transported in the anterograde direction at a rapid rate, the release of labeled phospholipid from cell bodies in the dorsal root ganglion is prolonged, continuing for about 1 week (Toews et al., 1983). The time course of retrograde axonal transport is consistent with these previously demonstrated anterograde transport kinetics. The peak of retrograde transport occurs later (4 to 10 days) and is more prolonged than that for anterograde transport ( 1 to 3 days). The temporal delay in peak retrograde accumulation relative to the peak of anterograde accumulation presumably reflects the time interval necessary for the transported material to reach the distal nerve terminals, undergo "turn-around," and return along the axon to the ligature. The retro-

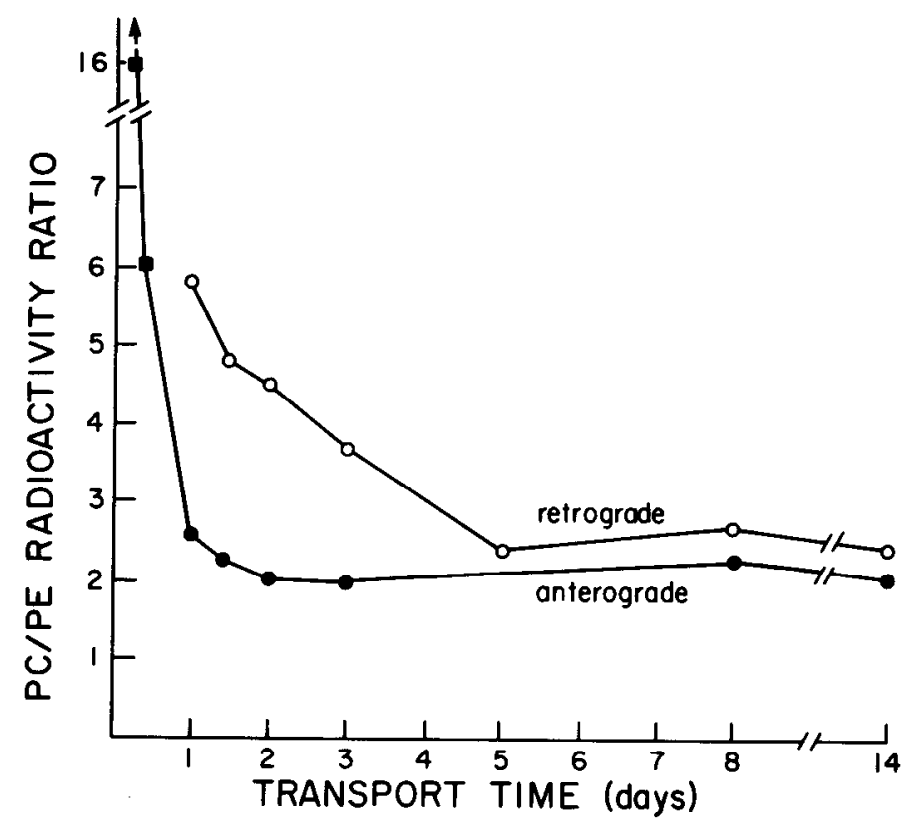

Figure 5. Changes with time in phosphatidylcholine to phosphatidylethanolamine $(P C / P E)$ radioactivity ratios in the retrograde accumulation segment and in the corresponding segment from the contralateral unligated nerve. At various times (noted on the abscissa as transport time) following injection of $\left[2-{ }^{3} \mathrm{H}\right]$ glycerol into both $\mathrm{L} 5$ dorsal root ganglia, ligatures were applied to the left sciatic nerve. After a $24-\mathrm{hr}$ collection time, PC/PE radioactivity ratios in the segment distal to the distal ligature $(O$, retrograde line) and in the corresponding segment from the unligated nerve $(\mathbf{O}$, anterograde line) were determined. The high PC/PE radioactivity ratio observed initially for the retrograde transport line is seen to mimic that in the material initially transported (anterograde line) with an appropriate temporal offset. Values for 6 and 12-hr anterograde points $(\square$ ) were obtained from a previous study (Toews et al., 1983).

grade transport peak may also be more prolonged than that for anterograde transport due to the arborization of the distal terminals at varying distances along the hindlimb, as well as the possibility that the "turn-around" time is not uniform at all of the nerve terminals. Due to the prolonged period of release from the cell bodies, labeled phospholipids do not form a discrete wave of material moving to the axons and nerve endings. For this reason, it is difficult to make distinct measurements of "turn-around" times for phospholipids, as can be done for rapidly transported proteins (Bisby and Bulger. 1977; Sahenk and Mendell, 1980). Our estimates suggest that the "turn-around" times for radioactive phospholipids may range from 12 hr to about 2 weeks, as judged from the studies of relative accumulation as a function of transport time.

The phospholipid class label distribution for both the retrograde and anterograde transport phases was also examined in order to gain further information about the relationship between lipids arriving at axons and nerve endings by anterograde transport, and those returning to the nerve cell bodies by retrograde transport. In the retrograde accumulation segment, labeled lipids from each of the major phospholipid classes were present in proportions similar to those seen at earlier times for anterograde transport. Phosphatidylcholine and phosphatidylethanolamine together accounted for 60 to $80 \%$ of the total retrograde accumulating lipid radioactivity, with phosphatidylserine and phosphatidylinositol accounting for most of the remainder. Changes with time in the phospholipid class radioactivity distribution in the retrograde accumulation segment were also similar to changes seen earlier for anterograde transport. The ratio of radioactivity in phosphatidylcholine relative to that in phosphatidylethanolamine in this segment was initially high (5.8 at 1 day), relative to later time points $(\sim 2.5)$. This presumably reflects the fact that labeled phospholipids initially reaching the axons and nerve endings by anterograde transport are also enriched in phosphatidyl- 
choline, relative to phosphatidylethanolamine (Toews et al., 1983) Thus, both the time course of transport and the temporal changes in phospholipid class label distribution for retrograde transport are similar to those for anterograde transport but are delayed by several days. We suggest that phospholipids delivered to nerve endings by anterograde transport enter a pool of lipids from which they are available for incorporation into the retrograde phase of axonal transport. The distribution of radioactivity among phospholipids committed to retrograde transport reflects the radioactivity distribution among phospholipids in the pool at that time.

\section{References}

Abe, T., H. Tatsuga, and M. Kurokawa (1973) Rapid transport of phosphatidylcholine occurring simultaneously with protein transport in the frog sciatic nerve. J. Biochem. 136: 731-740.

Alberghina, M., M. Viola, M. Moro, and A. M. Giuffrida (1981) Axonal transport of phospholipids in rabbit optic pathway. Neurochem. Res. 6: 633-647.

Alberghina, M., F. Moschella, M. Viola, V. Brancat, G. Micali, and A. M. Giuffrida (1983) Changes in rapid transport of phospholipids in the rat sciatic nerve during axonal regeneration. J. Neurochem. 40: 32-38.

Armstrong, R., R. Ray, A. D. Toews, and P. Morell (1984) Retrograde axonal transport of endogenous phospholipids. Trans. Am. Soc. Neurochem. 15: 247.

Benjamins, J. A., and G. M. McKhann (1973) $\left[2-{ }^{3} \mathrm{H}\right]$ Glycerol as a precursor of phospholipids in rat brain: Evidence for lack of recycling. J. Neurochem. 20: 1111-1120.

Benjamins, J. A., S. Miller, and P. Morell (1976) Metabolic relationships between myelin subfractions: Entry of galactolipids and phospholipids. J. Neurochem. 27: 565-570

Bisby, M. A. (1977) Retrograde axonal transport of endogenous protein: Differences between motor and sensory axons. J. Neurochem. 28: 249251.

Bisby, M. A. (1980) Retrograde axonal transport. In Advances in Cellular Neurobiology, L. Hertz and S. Federoff, eds., Vol. I, pp. 69-117, Acadmic Press, Inc., New York.

Bisby, M. A. (1982) Functions of retrograde axonal transport. Fed. Proc. 41: $2307-2311$

Bisby, M. A. (1983) Retrograde axonal transport. J. Neurochem. 41: S70.

Bisby, M. A., and V. T. Bulger (1977) Reversal of axonal transport at a nerve crush. J. Neurochem. 29: 313-320.

Brunetti, M., L. Di Giamberardino, G. Porcellati, and B. Droz (1981) Contribution of axonal transport to the renewal of myelin phospholipids in peripheral nerves. II. Biochemical study. Brain Res. 219: 73-84.

Cuenod, M., and J. Schonbach (1971) Synaptic proteins and axonal flow in the pigeon visual pathway. J. Neurochem. 18: 809-816.

Currie, J. R., B. Grafstein, M. H. Whitnall, and R. Alpert (1978) Axonal transport of lipid in goldfish optic axons. Neurochem. Res. 3: 479-492

Di Giamberardino, L., G. Bennett, H. L. Koenig, and B. Droz (1973) Axonal migration of protein and glycoprotein to nerve endings. III. Cell fraction analysis of chicken ciliary ganglion after intracerebral injection of labeled precursors of proteins and glycoproteins. Brain Res. 60: 147-159.

Droz, B., A. Rambourg, and H. L. Koenig (1975) The smooth endoplasmic reticulum: Structure and role in the renewal of axonal membrane and synaptic vesicles by fast axonal transport. Brain Res. 93: 1-3.

Droz, B., L. Di Giamberardino, and H. L. Koenig (1981) Contribution of axonal transport to the renewal of myelin phospholipids in peripheral nerves. I. Quantitative radioautographic study. Brain Res. 219: 57-71.

Elam, J. S., and B. W. Agranoff (1971) Rapid transport of protein in the optic system of the goldfish. J. Neurochem. 18: 375-387.

Folch, J., M. Lees, and G. H. Sloane-Stanley (1957) A simple method for the isolation and purification of total lipids from animal tissues. J. Biol. Chem. 226: 497-509.

Forman, D. S., A. L. Padjen, and G. R. Siggins (1977) Axonal transport of organelles visualized by light microscopy: Cinemicrographic and computer anałysis. Brain Res. 136: 197-213.

Gould, R. M., W. D. Spivac, R. S. Sinatra, T. O. Lindquist, and N. A. Ingolia (1982) Axonal transport of choline lipids in normal and regenerating rat sciatic nerve. J. Neurochem 39: 1569-1578.

Grafstein, B., and D. S. Forman (1980) Intracellular transport in neurons. Physiol. Rev. 60: 1167-1283.

Gratstein, B., J. A. Miller, R. W. Ledeen, J. Haley, and S. C. Specht (1975)
Axonal transport of phospholipids in goldfish optic system. Exp. Neurol. 46: $261-281$

Haley, J. E., L. J. Tirri, and R. W. Ledeen (1979) Axonal transport of lipids in the rabbit optic system. J. Neurochem. 32: 727-734.

Hoffman, P. N., and R. J. Lasek (1975) The slow component of axonal transport. Identification of major structural polypeptides of the axon and their generality among mammalian neurons. J. Cell Biol. 66: 351-366.

Horrocks, L. A., and G. Y. Sun (1972) Ethanolamine plasmalogens. In Research Methods in Neurochemistry, N. Marks and R. Rodnight, eds. Vol. I, pp. 223-231, Plenum Press, New York.

Kapeller, K., and D. Mayor (1969) Electron microscopic study of the early changes distal to a constriction in sympathetic nerves. Proc. R. Soc. Lond. (Biol.) 172: 53-63.

Karisson, J. O., and J. Sjostrand (1971) Synthesis, migration and turnover of protein in retinal ganglion cells. J. Neurochem. 18: 749-767.

Kristensson, K. (1982) Retrograde axonal transport of exogenous macromolecules. In Axoplasmic Transport, D. G. Weiss, ed., pp. 200-205, Springer-Verlag, New York

Ledeen, R. W., and J. E. Haley (1983) Axon-myelin transfer of glycerollabeled lipids and inorganic phosphate during axonal transport. Brain Res. 269: $267-275$.

Levine, J., and M. Willard (1980) The composition and organization of axonally transported proteins in the retinal ganglion cells of the guinea pig. Brain Res. 194: 137-154.

Longo, F. M., and R. Hammerschlag (1980) Relation of somal lipid synthesis to the fast axonal transport of protein and lipid. Brain Res. 193: 471-485.

Miani, N. (1962) Evidence of a proximo-distal movement along the axon of phospholipid synthesized in the nerve-cell body. Nature 193: 887-888.

Miller, S. L., J. A. Benjamins, and P. Morell (1975) Reutilization of choline for synthesis of phosphatidylcholine and choline plasmalogen in rat brain, $\mathrm{J}$. Neurochem. 25: 913-914.

Ochs, S. (1983) Axoplasmic transport. In Handbook of Neurochemistry. Vol. V: Metabolic Turnover in the Nervous System, A. Lajtha, ed., pp. 355379, Plenum Press, New York.

Rambourg, A., and B. Droz (1980) Smooth endoplasmic reticulum and axonal transport. J. Neurochem. 35: 16-25.

Sahenk, Z., and J. R. Mendell (1980) Axoplasmic transport in zinc pyridinethione neuropathy: Evidence for an abnormality in distal turn-around. Brain Res. 186: 343-353.

Schwab, M. E., and H. Thoenen (1983) Retrograde axonal transport. In Handbook of Neurochemistry. Vol. V: Metabolic Turnover in the Nervous System, A. Lajtha, ed., pp. 381-404, Plenum Press, New York.

Schwartz, J. H. (1979) Axonal transport: Components, mechanisms, and specificity. Annu. Rev. Neurosci. 2: 467-504.

Sjostrand, J. (1979) Fast and slow components of axoplasmic transport in the hypoglossal and vagus nerves of the rabbit. Brain Res. 18: 461-467.

Smith, R. S. (1980) The short term accumulation of axonally transported organelles in the region of localized lesions of single myelinated axons. $\mathrm{J}$. Neurocytol. 9: 39-65

Thoenen, H., and M. E. Schwab (1978) Physiological and pathophysiological implications of the retrograde axonal transport of macromolecules. Adv. Pharmacol. Chemother. 5: 37-59.

Toews, A. D., and P. Morell (1981) Turnover of axonally transported phospholipids in nerve endings of retinal ganglion cells. J. Neurochem. 37: 1316-1323.

Toews, A. D., J. F. Goodrum, and P. Morell (1979) Axonal transport of phospholipids in rat visual system. J. Neurochem. 32: 1165-1173.

Toews, A. D., S. S. Padilla, L. J. Roger, and P. Morell (1980) Axonal transport of glycerophospholipids following intracerebral injection of glycerol into substantia nigra or lateral geniculate body. Neurochem. Res. 5: 11751183.

Toews, A. D., B. F. Saunders, W. D. Blaker, and P. Morell (1983) Differences in the kinetics of axonal transport for individual lipid classes in rat sciatic nerve. J. Neurochem. 40: 555-562.

Tsukita, S., and H. Ishikawa (1980) The movement of membranous organelles in axons: Electron microscopic identification of anterogradely and retrogradely transported organelles, J. Cell Biol. 84: 513-530.

Tytell, M., M. M. Black, J. Garner, and R. J. Lasek (1981) Axonal transport: Each of the major rate components consists of distinct macromolecular complexes. Science 214: 179-181.

Weiss, D. G. (1982) Axoplasmic Transport, Springer-Verlag, New York.

Willard, M., M. W. Cowan, and P. R. Vagelos (1974) The polypeptide composition of intra-axonally transported proteins: Evidence for four transport velocities. Proc. Natl. Acad. Sci. U. S. A. 71: 2182-2187 